\title{
Evolution of energy in flow driven by rising bubbles
}

\author{
Irene M. Mazzitelli* and Detlef Lohse ${ }^{\dagger}$ \\ Physics of Fluids Group, Impact Institute, Department of Science and Technology, J. M. Burgers Centre for Fluid Dynamics, \\ University of Twente, P.O. Box 217, 7500 AE Enschede, The Netherlands
}

(Received 7 April 2008; revised manuscript received 7 January 2009; published 30 June 2009)

\begin{abstract}
We investigate by direct numerical simulations the flow that rising bubbles cause in an originally quiescent fluid. We employ the Eulerian-Lagrangian method with two-way coupling and periodic boundary conditions. In order to be able to treat up to 288000 bubbles, the following approximations and simplifications had to be introduced, as done before, e.g., by Climent and Magnaudet, Phys. Rev. Lett. 82, 4827 (1999). (i) The bubbles were treated as point particles, thus (ii) disregarding the near-field interactions among them, and (iii) effective force models for the lift and the drag forces were used. In particular, the lift coefficient was assumed to be 1/2, independent of the bubble Reynolds number and the local flow field. The results suggest that large-scale motions are generated, owing to an inverse energy cascade from the small to the large scales. However, as the Taylor-Reynolds number is only in the range of 1 , the corresponding scaling of the energy spectrum with an exponent of $-5 / 3$ cannot develop over a pronounced range. In the long term, the property of local energy transfer, characteristic of real turbulence, is lost and the input of energy equals the viscous dissipation at all scales. Due to the lack of strong vortices, the bubbles spread rather uniformly in the flow. The mechanism for uniform spreading is as follows. Rising bubbles induce a velocity field behind them that acts on the following bubbles. Owing to the shear, those bubbles experience a lift force, which makes them spread to the left or right, thus preventing the formation of vertical bubble clusters and therefore of efficient forcing. Indeed, when the lift is artificially put to zero in the simulations, the flow is forced much more efficiently and a more pronounced energy that accumulation at large scales (due to the inverse energy cascade) is achieved.
\end{abstract}

DOI: 10.1103/PhysRevE.79.066317

PACS number(s): 47.55.Kf, 47.55.Ca

\section{INTRODUCTION}

Bubbly flows are ubiquitous in nature and technology, but exact analytical or numerical results are extremely difficult to obtain due to the multiscale nature of the problem and due to the moving interfaces. For a review on the numerical modeling, we refer to $[1,2]$, for recent reviews on the experimental situation we refer to $[3,4]$, and our own recent work on the subject has been summarized in [5]. An excellent overview on the experimental, numerical, and theoretical knowledge for various bubble Reynolds numbers can also be found in Refs. [6,7].

The motion of the small bubbles in the fluid induces velocity fluctuations that can be either dissipated immediately by viscosity or can be enhanced, thus, generating motion on scales much larger than the disturbance dimension. Owing to their random character, these fluctuations are referred to as "pseudoturbulence" $[4,6,8]$. In a flow initially at rest and only forced by rising bubbles, the pseudoturbulent fluctuations are the only source of energy. Otherwise, they can add to the already existing fluid velocity fluctuations, which are driven in some other way. Depending on the flow conditions and on the bubble size distribution, the turbulent energy dissipation may be either enhanced or suppressed.

Lance and Bataille [9] suggested that the effect of bubbles on the flow depends on the ratio of kinetic energy due to the bubble motion and the typical kinetic energy $\left\langle u^{\prime 2}\right\rangle$ of the

\footnotetext{
*Present address: Istituto de Sciencze dell'Atomosfera e del Clima, CNR, via del Fosso del Cavaliere, 100, 00133 Rome, Italy.

†Email: d.lohse@utwente.nl
}

fluctuations in the liquid velocity before bubble injection,

$$
b=\frac{1}{2} \alpha v_{T}^{2} /\left\langle u^{\prime 2}\right\rangle,
$$

where $u^{\prime}$ is the typical flow velocity fluctuation, $\alpha$ is the void fraction, $v_{T}$ is the bubble rise velocity in still water, and we have taken $\frac{1}{2}$ for the added mass coefficient. The ratio $b$ is called the bubblance parameter [5]. For $b \ll 1$, the kinetics of the bubbly flow are entirely dominated by the turbulent energy of the flow and the bubbles can be considered as some distortion, such as in the experiments of Refs. [10-15] or in the numerical simulations of Refs. [16-21]. In contrast, for $b \gg 1$, the flow is dominated by the bubble motion. In this case, we have bubblance rather than turbulence, such as in the experiments of Refs. [4,9,22-25] or in the numerical simulations of Refs. [6,7,26-29]. The analogous situation for sedimenting particles was experimentally analyzed by Faeth and co-workers, both for particles in water [30] and in air [31].

In the present work, we focus on the bubblance case $b$ $\gg 1$, namely, on microbubbles rising in an initially quiescent flow, where formally $b=\infty$. These conditions imply that pseudoturbulence, due to the bubble buoyancy, is the only source of flow energy; thus, bubbles drive the turbulence and eventually the energy dissipation. We will address the following questions: (i) what is the time evolution of the energy of bubbly driven turbulence initially at rest?; (ii) are microbubbles able to induce in still fluid a flow that possesses similar features as real turbulence, i.e., can the inertial scaling law characteristic of homogeneous and isotropic turbu- 
lence be attained in a flow forced solely by bubbles?; and (iii) how are bubbles eventually distributed in such flow and what forces determine this distribution?

To be able to address these questions, the flow should best be driven at least by ten thousands of bubbles, in order to have sufficient statistics. While hundreds of bubbles can still be treated with front-tracking techniques [6,7,27-29,32], resolving both the shape of the bubble and the flow around it, this clearly is no longer possible for ten thousands of bubbles and one therefore is forced to employ approximations. We will thus follow the complementary approach and model the bubbles as point particles, on which effective forces such as drag and lift act [1], similarly as was also done by Climent and Magnaudet [26]. We thus disregard the near-field interactions among the bubbles. Also the effective forces had to be modeled, namely, by choosing drag and lift coefficients. Unfortunately, the lift coefficient is not well known [1]. In fact, it depends on the bubble Reynolds number and on the local shear and vorticity in a highly nontrivial way [33-38]. Moreover, due to the interactions with the wake, effective forces on bubbles can depend on the bubble concentration and can even be nonlocal in time (history forces) $[33,39,40]$. Given these complications, for conceptional simplicity, we decided to simply use the Auton lift coefficient $C_{L}=1 / 2$ [41], realizing that at best qualitative agreement between our simulations and possible experiments can be expected. Finally, periodic boundary conditions are employed in all directions, including the vertical one. This means that bubbles leaving the periodic box at the top enter it back at the bottom, inducing a mean vertical flow.

Summarizing the various limitations and approximations, which were necessary in the numerical simulations, the aim of the present paper can only be to identify mechanisms; no quantitative predictions or comparisons with experiments are possible. The numerical simulations for bubble-induced convection [26] or for Taylor-Couette flow with microbubbles inducing drag reduction [42] were done in the same spirit with related numerical schemes and, indeed, the relevant physical mechanisms could be identified.

Our numerical simulations are based on the code extensively described in [17], but now as stated above with forcing only by the bubbles to mimic the pseudoturbulent flow. For completeness, we briefly repeat the dynamical equations and the central assumption in Sec. II. Sections III and IV describe the time evolution of global and spectral observables, respectively. In Sec. V we propose a qualitative physical explanation for the detected fluid energy time evolution. Section VI contains conclusions.

\section{BUBBLES AND FLUID EQUATIONS}

\section{A. Bubble motion equation}

The motion of a small undeformable bubble embedded in a velocity field $\mathbf{u}(\boldsymbol{x}, t)$ can be modeled by the equation (see, e.g., $[1,26,43])$,

$$
\begin{aligned}
\frac{d \mathbf{v}}{d t}= & 3 \frac{D \mathbf{u}}{D t}+\frac{1}{\tau_{b}}\{\mathbf{u}[\boldsymbol{y}(t), t]-\mathbf{v}(t)\}-2 \mathbf{g}-2 C_{L}\{\mathbf{v}(t) \\
& -\mathbf{u}[\boldsymbol{y}(t), t]\} \times \boldsymbol{\omega}[\boldsymbol{y}(t), t] .
\end{aligned}
$$

The four terms on the right-hand side represent (i) fluid ac- celeration plus added mass, (ii) drag, (iii) buoyancy, and (iv) lift, where we have explicitly written out the lift coefficient $C_{L}$, which-as discussed in the introduction-will be taken to be $C_{L}=1 / 2$. We will, however, also compare the simulations with those for $C_{L}=0$. The various symbols denote $\boldsymbol{y}(t)$ as the bubble position, $\mathbf{v}$ as the bubble velocity, $\boldsymbol{\omega}=\boldsymbol{\nabla} \times \mathbf{u}$ as the fluid vorticity, $\mathbf{g}$ as the gravity (acting in negative $z$ direction), and $\tau_{b}$ as the relaxation time, i.e., the time needed to adjust the bubble velocity to that of the fluid. The latter is related with the terminal velocity $v_{T}$ in still fluid $v_{T}=2 g \tau_{b}$. In the case of small bubble Reynolds number $\operatorname{Re}=2|\mathbf{u}-\mathbf{v}| a / \nu$ $<1$, with $a$ as the bubble radius and $\nu$ as the kinematic viscosity, for the bubble response time it holds $\tau_{b}=a^{2} / 6 \nu$ $[44,45]$. For larger bubbles, the prefactor in this relation is larger than $1 / 6$, which, however, would only quantitatively affect our results. The material derivative $(D / D t)$ of the fluid velocity is evaluated at the bubble position. We refer to Refs. $[1,17,46]$ for a derivation of the various terms in Eq. (2).

\section{B. Simulation of the flow}

The computational domain is a cube of side $L_{0}=2 \pi$, consisting of $N^{3}=128^{3}$ mesh points and subjected to periodic boundary conditions. The simulation is started at $t=0$ with the flow at rest and with $N_{b}$ bubbles with $\operatorname{Re} \sim O(1)$ placed at random locations. Bubbles rise because of gravity and transfer momentum to the fluid. We track their trajectories and we treat each bubble as a point source of momentum. Then, the total action on the flow results by summing the $\delta$ forcing that the bubbles apply at their positions $[17,26,47]$,

$$
\mathbf{f}_{b}(\boldsymbol{x}, t)=\sum_{i=1}^{N_{b}}\left[\frac{D}{D t} \mathbf{u}\left[\boldsymbol{y}_{i}(t), t\right]-\mathbf{g}\right]\left(\frac{4 \pi}{3} a^{3}\right) \delta\left[\boldsymbol{x}-\boldsymbol{y}_{i}(t)\right] .
$$

Here $\mathbf{g}$ is the gravity directed along the negative $z$ axis. The induced flow velocity $\mathbf{u}(\boldsymbol{x}, t)$ evolves according to the incompressible Navier-Stokes equation

$$
\frac{\partial \mathbf{u}}{\partial t}+\mathbf{u} \cdot \nabla \mathbf{u}=-\nabla p+\nu \Delta \mathbf{u}+\mathbf{f}_{b},
$$

which is solved by direct numerical simulation. The coupling of the bubbles toward the liquid is thus achieved by introducing the bubble forcing (3) into the Navier-Stokes equation (4) at the positions of the bubbles. Those are given by the dynamical equation (2) for the bubble position.

We use the pseudospectral code described in [17], where also the other details on the numerical simulations can be found. The point force approximation is validated by performing the same tests as in [17]. We stress that in contrast to that earlier work of ours, here Eq. (4) does not contain any forcing on the large scales. The flow is sustained solely by the bubble forcing term $\mathbf{f}_{b}$.

We analyze different cases. A list of the flow and bubbles parameters is shown in Table I. In Table II, the values of the numerical parameters and their physical equivalents are presented for some of the simulations performed.

\section{EVOLUTION IN TIME FOR GLOBAL QUANTITIES}

We describe the energy time evolution of the pseudoturbulent field generated by the rise of microbubbles. As al- 
TABLE I. Simulation parameters for all cases analyzed: void fraction $\alpha$, total bubbles number $N_{b}$, bubble Reynolds number $\operatorname{Re}=2 v_{T} a / \nu$, bubble response time $\tau_{b}$, equivalent radius $a$ in physical units, potential flow estimate of the total energy induced in the flow $E_{p}=\alpha v_{T}^{2} / 4$, and time asymptotic estimate from our calculations of the total energy $E$. This value has been determined from time averaging the statistically stationary state. In the numerical simulations, the kinematic viscosity $\nu=0.007, \alpha$, the rise speed in still fluid $v_{T}$ $=0.578$, and $\tau_{b}$ are fixed; the other quantities result consequently. In particular, the intensity of the gravity results from $g=v_{T} / 2 \tau_{b}$ and therefore is different in the simulation (d) as compared to the other ones.

\begin{tabular}{|c|c|c|c|c|c|c|c|}
\hline & $\alpha$ & $N_{b}$ & $\operatorname{Re}$ & $\tau_{b}$ & $\begin{array}{c}a \\
(\mu \mathrm{m})\end{array}$ & $E_{p} \times 10^{3}$ & $E \times 10^{3}$ \\
\hline (a) & $1.6 \%$ & 144000 & 3.10 & $8.4 \times 10^{-3}$ & 78 & 1.34 & 1.96 \\
\hline (b) & $0.8 \%$ & 72000 & 3.10 & $8.4 \times 10^{-3}$ & 78 & 0.67 & 1.38 \\
\hline (c) & $3.2 \%$ & 288000 & 3.10 & $8.4 \times 10^{-3}$ & 78 & 2.67 & 2.25 \\
\hline (d) & $1.6 \%$ & 50848 & 4.37 & $16.7 \times 10^{-3}$ & 87 & 1.34 & 1.57 \\
\hline
\end{tabular}

ready stated, at time $t=0$, the bubbles are randomly placed in the flow, that is originally at rest. Their rise displaces liquid and thus generates velocity fluctuations. If these fluctuations are not rapidly dissipated by viscosity, they can be transmitted to larger scales. As a consequence, large-scale motions are produced and the flow may become turbulent.

We investigate this issue by measuring average flow quantities as well as by studying the spectral energy distribution. We focus on case (a) of Table I.

In Fig. 1(a) we plot the total fluid energy $E(t)=\left\langle u_{x}(t)^{2}\right.$ $\left.+u_{y}(t)^{2}+u_{z}(t)^{2}\right\rangle / 2$, as a function of time. In the beginning, $E(t)$ undergoes a steep rise, afterward it slowly decreases, until it begins to oscillate and a statistically stationary state is reached. The behavior qualitatively resembles that one of the front-tracking simulations of Ref. [6] (see Fig. 1 of that paper). The kinetic energy is mainly generated by the momentum transfer in the direction of gravity, as we confirm by measuring the three components of the fluid velocity fluctuations $\left\langle u_{i}^{2}\right\rangle$, with $i=x, y, z$, which are much larger in the $z$ direction than in the horizontal ones, and the unidimensional Taylor-Reynolds number, defined as

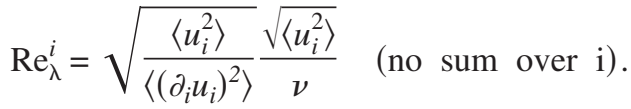

The behavior of $\operatorname{Re}_{\lambda}^{i}$ as function of time is presented in Fig. 2. It is evident from the plot that the flow displays strong asymmetry due to the vertical driving through the rising bubbles and that there is a mean flow in the vertical direction. However, it is also clear that due to the dissipation, both

TABLE II. Simulation parameters for cases (a)-(c) and corresponding physical equivalents. The bubble Reynolds number is $\mathrm{Re}=2 v_{T} a / \nu=3.10$.

\begin{tabular}{lcc}
\hline \hline & Dimensionless parameter & Physical equivalent \\
\hline$\nu$ & 0.007 & $10^{-2} \mathrm{~cm}^{2} / \mathrm{s}$ \\
$g$ & 34.55 & $981 \mathrm{~cm} / \mathrm{s}^{2}$ \\
$a$ & 0.019 & $78 \mu \mathrm{m}$ \\
$v_{T}$ & 0.58 & $2 \mathrm{~cm} / \mathrm{s}$ \\
$\tau_{b}$ & $8.4 \times 10^{-3}$ & $1 \mathrm{~ms}$ \\
\hline \hline
\end{tabular}

the energy and the vertical velocity remain bounded, in spite of the periodic boundary conditions. In by passing, we note the interesting analogy with the so-called homogenous Rayleigh-Bénard convection [48], where the flow is also periodic in all directions, with a vertical volume forcing. In Ref. [49], we have shown that though in that case exponentially growing solutions exist, they are subject to instabilities that limit their growth to produce statistically steady flow.

We now compare the results on the energies in the present pseudoturbulence simulations with potential flow theory, see, e.g., the work of van Wijngaarden $[8,50,51]$. We do not expect agreement given that we deal with point particles and that the potential flow results of Refs. [8,50,51] only hold in the high bubble Reynolds number case, and here we have $\mathrm{Re}$ between 3 and 5 . Nonetheless, we consider this comparison to be instructive and, surprisingly, we find the saturated kinetic energy to be on the order of $\alpha v_{T}^{2} / 4$, in agreement with the potential flow theory results for high Re bubbles. However, the redistribution of the energy along the three directions deviates from what is predicted by the potential flow analysis, according to which we should have [50],

$$
\left\langle u_{z}^{2}\right\rangle \simeq \frac{1}{5}\left(\alpha v_{T}^{2}\right), \quad\left\langle u_{x}^{2}\right\rangle=\left\langle u_{y}^{2}\right\rangle \simeq \frac{3}{20}\left(\alpha v_{T}^{2}\right) .
$$

The potential flow value is $\left\langle u_{z}^{2}\right\rangle /\left\langle u_{x}^{2}\right\rangle \simeq 4 / 3$, whereas in our simulation this ratio is about 15 . In the opposite limit $\mathrm{Re}$ $\rightarrow 0$, under Stokes flow condition, the fluid equations are linear. In this regime, symmetry considerations require that rising bubbles cannot force the flow in directions perpendicular to their motion (provided that there are no walls but periodic boundary conditions as in our case): a result that is also intuitive for rising point particles in fluid at rest. As a consequence, the ratio $\left\langle u_{z}^{2}\right\rangle /\left\langle u_{x}^{2}\right\rangle \rightarrow \infty$. Our result, for small but finite Reynolds number, lies in between the two limits discussed. The same holds for the case of sedimenting particles in water (with larger particle Reynolds numbers between 38 and 545), where this ratio is between 4 and 25 [30].

The total energy induced by high Re bubbles, for which inertia effects are dominant, can be easily estimated by the following argument. At low void fractions, the flow energy is the sum of the energy induced by individual bubbles, i.e., $E \simeq N_{b}(1 / 2) m_{b} v_{T}^{2}$, where the effective mass of a bubble is 

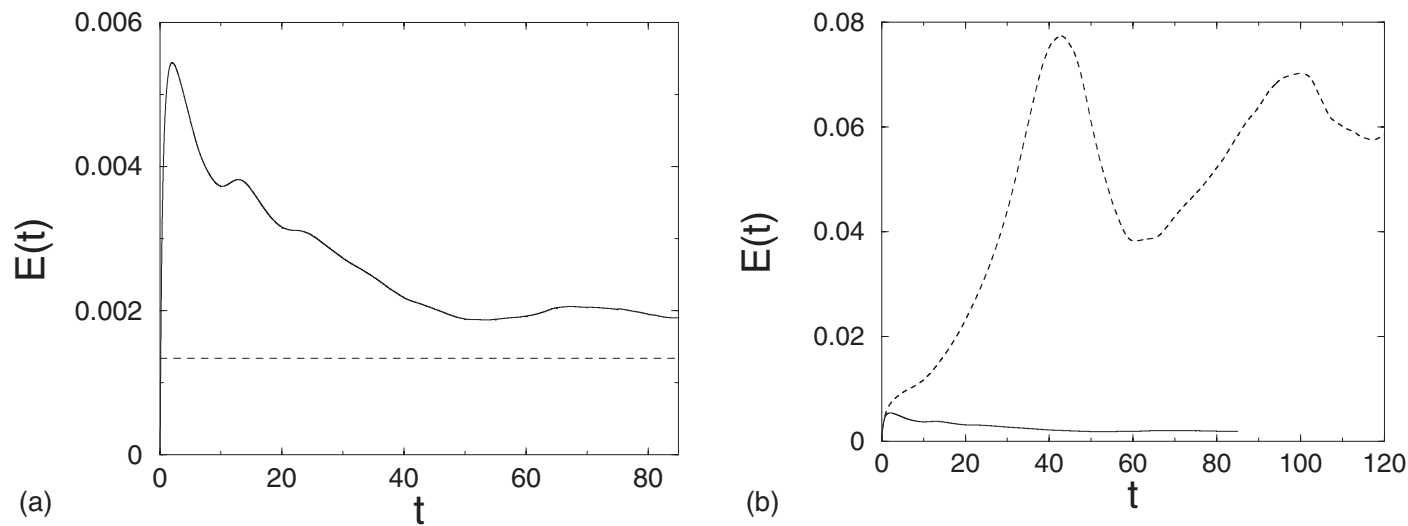

FIG. 1. (a) Total fluid energy as a function of time. The straight dashed line indicates the potential flow result $E=\alpha v_{T}^{2} / 4$. (b) Total fluid energy in simulation with (solid line) and without the lift (dashed line) as functions of time.

$m_{b}=\rho_{f}\left(2 \pi a^{3} / 3\right)$, owing to the added mass factor $1 / 2$. Thus, $E \simeq \alpha v_{T}^{2} / 4$. On the other hand, when $\operatorname{Re} \sim 1$, to estimate the total energy induced by sedimenting particles or rising bubbles is far more complicated. Indeed, for $\mathrm{Re} \ll 1$, the flow induced by one particle decreases, with the distance $r$ from the particle — as $1 / r$ - thus, leading to a total flow energy that diverges with the system size. Different screening mechanisms have been invoked in the past in order to account for this problem (see, e.g., [52-57]), but this issue is beyond the scope of the present paper.

In Table I we report the total energy estimated in our simulations, correspondent to different void fractions and bubble dimensions. In all cases, the energy induced is on the order of $\alpha v_{T}^{2} / 4$. We note by comparing cases (a) and (d), that when increasing the bubble dimension while fixing the void fraction, thus reducing the total bubble-fluid interface, less energy is generated in the flow.

\section{EVOLUTION IN TIME FOR SPECTRA}

After transforming to wave-number space, we consider the time development of the energy spectrum,

$$
E(k, t)=\frac{1}{2} \sum_{k<|\mathbf{k}|<k+d k} u_{i}^{*}(\mathbf{k}, t) u_{i}(\mathbf{k}, t) \quad i=x, y, z .
$$

Here $u_{i}(\mathbf{k}, t)$ is the $i$ th component of the fluid velocity in $k$ space and repeated indices are considered summed. $E(k, t)$ is

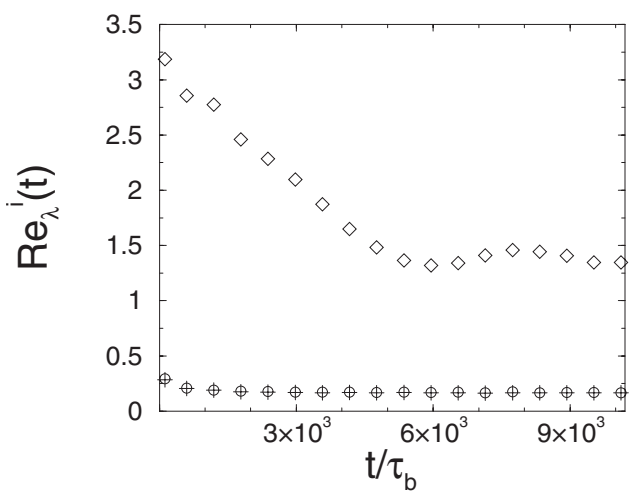

FIG. 2. Behavior of the unidimensional Taylor-Reynolds number as function of time, in the $x$ (pluses), $y$ (circles), and $z$ (diamonds) directions. the total energy contained in a spherical shell of radius $k$ and width $d k$.

In Fig. 3(a), the energy spectrum averaged on four subsequent time intervals is presented. The intervals correspond to $0<t / \tau_{b}<6 \times 10^{2}, \quad 12 \times 10^{2}<t / \tau_{b}<18 \times 10^{2}, \quad 24 \times 10^{2}$ $<t / \tau_{b}<30 \times 10^{2}$, and $100 \times 10^{2}<t / \tau_{b}<106 \times 10^{2}$ in Fig. 1 . The figure depicts that the energy is originally introduced at high wave numbers and gradually transported to larger scales (solid line). However, after some time the spectrum flattens and a nearly constant energy is measured at all scales. Thus, in the first stage of bubble-fluid coupling, an inverse energy cascade, from the small to the large scales, builds up largescale eddies. The corresponding slope of the spectrumindicating the local transfer of energy-would be $-5 / 3$; however, as the Taylor-Reynolds numbers are small, such a scaling regime cannot really develop. Later on, the inverse energy cascade cannot be sustained and it disappears in the final state. We investigate whether this last state is statistically stationary by having a closer look at the energy-transfer equation in $k$ space,

$$
\frac{\partial}{\partial t} E(k, t)=T(k, t)-D(k, t)+F_{b}(k, t) .
$$

Here, the various terms indicate, respectively, the energy transfer to wave number $k, T(k, t)$,

$$
T(k, t)=\sum_{k<|\mathbf{k}|<k+d k} T(\mathbf{k}, t),
$$

where

$$
T(\mathbf{k}, t)=\operatorname{Im}\left[k_{j} u_{l}^{*}(\mathbf{k}, t) \sum_{\mathbf{k}^{\prime}} u_{j}\left(\mathbf{k}-\mathbf{k}^{\prime}, t\right) u_{l}\left(\mathbf{k}^{\prime}, t\right)\right],
$$

the viscous dissipation, $D(k, t)=2 \nu k^{2} E(k, t)$, and the bubble forcing contribution $F_{b}(k, t)$,

$$
F_{b}(k, t)=\sum_{k<|\mathbf{k}|<k+d k} F_{b}(\mathbf{k}, t)
$$

where 

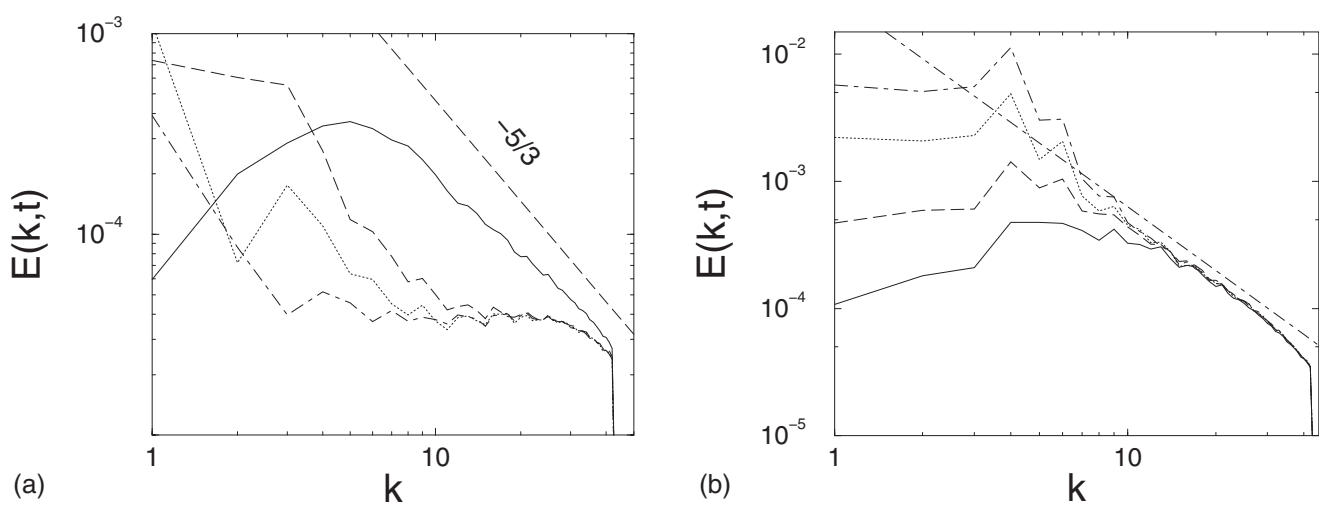

FIG. 3. (a) Energy spectra for the simulation that includes lift forces obtained by averaging on four different time intervals: $0<t / \tau_{b}$ $<6 \times 10^{2}$ (solid line), $12 \times 10^{2}<t / \tau_{b}<18 \times 10^{2}$ (dashed line), $24 \times 10^{2}<t / \tau_{b}<30 \times 10^{2}$ (dotted line), and $100 \times 10^{2}<t / \tau_{b}<106 \times 10^{2}$ (dot-dashed line). The straight line indicates the behavior in homogeneous and isotropic turbulence. The employed time period is a compromise of, on one hand, having sufficient statistics and, on the other hand, being able to follow the time evolution (see Fig. 1). We also tried slightly larger and smaller time periods and get similar results as those shown in this figure. So our results are robust. (b) Energy spectra for the simulation without lift forces obtained by averaging on four different time intervals. For the various symbols, look at the caption of (a).

$$
F_{b}(\mathbf{k}, t)=\operatorname{Re}\left[\mathbf{u}^{*}(\mathbf{k}, t) \cdot \tilde{\mathbf{f}}_{b}(\mathbf{k}, t)\right],
$$

with $\tilde{\mathbf{f}}_{b}(\mathbf{k}, t)$ as the Fourier transform of the coupling term $\mathbf{f}_{b}(\boldsymbol{x}, t)$, defined in Eq. (3). Here Im and Re indicate, respectively, the imaginary and real parts of the expression between the brackets.

The spectra of the bubble forcing and of the dissipation are shown in Fig. 4(a). The strongest forcing is concentrated on the small scales, as we expect, owing to the dimension of the energy sources. However, the energy that is initially transferred to the large scales via the nonlinear interactions has to return to the small scales in order to be dissipated by viscosity. In fact, there is no energy sink on the large scales that could take the energy out of the system. Therefore, the condition for establishing a stationary state is that the time average energy transfer has to be zero on all wave numbers and dissipation has to equal bubble forcing, i.e., $T(k)=0$ and $D(k)=F_{b}(k)$, where the time dependence has dropped out after the average on time.

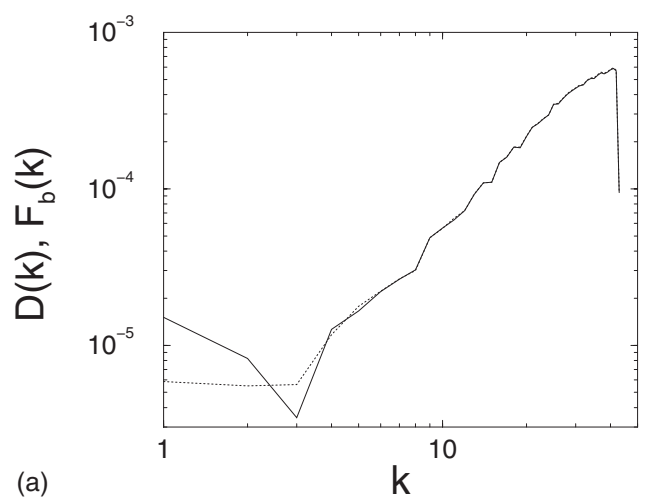

As we show in Fig. 4(a), apart from the large scales where the average still has not converged, this requirement is satisfied by our simulation. In real flow, with walls instead of periodic boundary conditions, energy dissipation in the developing boundary layers will of course, eventually, play a crucial role in the energy balance.

The time evolution of the energy spectrum can be compared to the one presented by [58], where the authors study a similar system, namely, fluid motion generated by rising bubbles, by applying a different technique for the implementation of two-way coupling. The results agree qualitatively, i.e., the initial induction of structures at large scale is followed by a state in which the slope of the energy spectrum is reduced. As the authors state themselves, the reason is connected with the temporal evolution of the bubble distribution. Indeed, bubble clusters, which are assembled in the beginning and are able to force the liquid efficiently, are not stable and bubbles tend to distribute uniformly in the flow. Moreover, the structures induced in the flow itself are far too weak to trap the bubbles. Thus, the phenomenon of vortex trapping

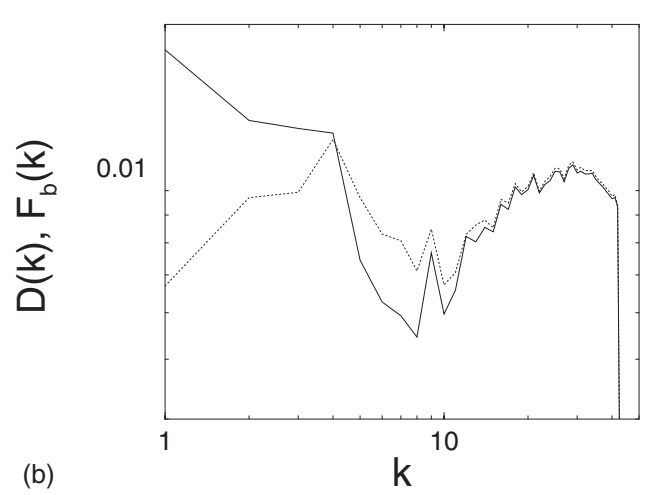

FIG. 4. (a) Time average of the contribution of the bubble forcing to the energy spectrum, as defined in Eq. (8) (solid line), and of the viscous energy dissipation $D(k)=2 \nu k^{2} E(k)$ (dotted line), in the simulation with the lift force. (b) Time average of the contribution of the bubble forcing to the energy spectrum (solid line) and of the viscous energy dissipation $D(k)=2 \nu k^{2} E(k)$ (dotted line), in the simulation without the lift force. 


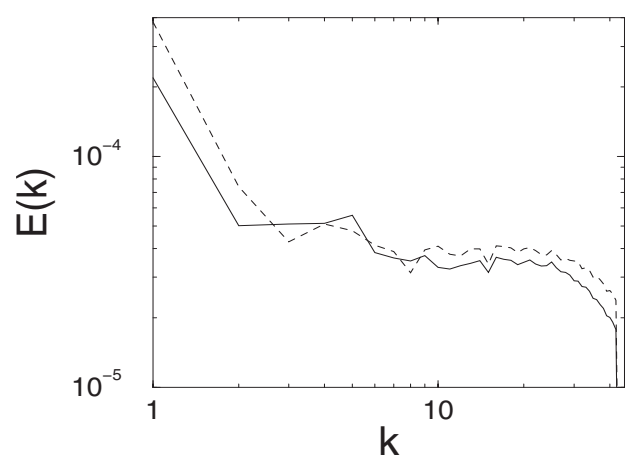

FIG. 5. Energy spectra, in the statistically stationary state, for case (a) (dashed line) and (d) (solid line) of Table I.

of bubbles does not occur and therefore high local bubble concentration as in bubbly turbulent flows (see, e.g., $[16,59,60])$ are not created here.

Note that the front-tracking simulations of Ref. [6] for 27 bubbles with a bubble Reynolds number of about 25 gave a quite different spectrum, namely, a slope of about -3.6 in the large wave-number regime. Also Martinez et al. [61] found a slope of -3.2 in the large wave-number regime of experimental velocity spectra in experimental pseudoturbulence generated with mm-size bubbles. Indeed, a slope -3 has theoretically been attributed [9] to this regime, in which the energy deposited by the wake is directly dissipated and which obviously cannot be modeled with the point-particle approach. All this wake energy dissipation is missing in our approach.

We carry on the comparison with the results of [58] by looking at the high wave-numbers behavior of the spectrum. In that paper, a steeper slope with respect to homogeneous and isotropic turbulence is observed. The critical wave number $k_{c}$ above which it shows up is estimated by the average distance between the bubbles, that is $L_{c} \sim 2 \pi / N_{b}^{1 / 3}$. In our simulation, for case (a) of Table I, we have $L_{c} \sim 0.12$ (about $1 / 50$ of the box with $L_{0}=2 \pi$ ), thus $k_{c}=2 \pi / L_{c} \sim 52$ and for case (d) $L_{c} \sim 0.17$, thus $k_{c} \sim 37$. As we show in Fig. 5, a transition in the slope of the energy spectrum occurs at high wave numbers. However, neither the critical wave number nor the slope of the spectra can be clearly defined.

We find agreement with the results of [58] on the strongly anisotropic energy distribution along the three velocity components. Indeed, also in that work, about the $90 \%$ of the flow energy is contained in the vertical component $(z)$ of the fluid velocity.

We again also compare our results with the case for sedimenting particles in originally still fluid [30,31]. Also for that case, the energy spectrum is very broad. The spectral slope of the frequency spectrum was found to be around -1.1 for remarkably several decades; but a comparison of this value with the slopes in the wave-number spectra obtained in this paper is difficult as Taylor's frozen flow hypothesis will most likely not hold for this weak turbulence.

\section{PHYSICAL EXPLANATION OF THE RESULTS}

The occurrence of the inverse cascade phenomenon in three-dimensional turbulence has been related to the pres-

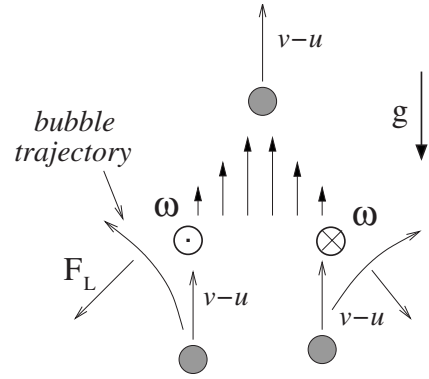

FIG. 6. Sketch of the action of the lift force on a bubble rising in the wake of another one: the lift tends to expel the bubble from the wake.

ence of strong anisotropies at small scales (see e.g., $[62,63]$ ). These anisotropies can be produced by bubble clusters elongated in the gravity direction. Within them, the energy production term due to the bubbles can be far more intense in the vertical direction than in the horizontal ones, owing to the high values reached by the $\langle\mathbf{u} \cdot \mathbf{g}\rangle$ contribution. The stability of these structures is opposed by a horizontal force that laterally spread the bubbles. When considering the bubble motion equation, it appears that the lift is the most relevant of such forces. Therefore, we further investigate the system by comparing the outcome of simulations with lift and without lift force.

Surprisingly, without the lift the results are very different. In Fig. 1(b), the total energy in the two simulations, one including the lift (solid line) and the other excluding it (dashed line), are compared. In both cases, the bubbles parameters correspond to run (a) in Table I. The energy induced in the second simulation is up to 30 times larger than in the first. The reason for the larger total energy (not necessarily the fluctuations) is illustrated in the sketch of Fig. 6. Without the lift, the rising bubbles can better group together and thus more efficiently force the flow; whereas with the lift, they are horizontally repelled from each other. Also Murai et al. [64] measured a higher turbulence intensity in numerical simulations without the lift force than in simulations with the lift, though the difference detected is quantitatively much smaller than in our case.

The behavior in spectral space is remarkably different too. In Fig. 3(b), the time evolution of the energy spectrum in the latter simulation is presented. The spectrum is averaged on four subsequent time intervals, which are the same as for Fig. 3(a). The process of inverse energy cascade is now strongly enhanced. In fact, the spectral intensity at small wave numbers increases in time, whereas it is constant at large ones.

Moreover, it is remarkable that a local slope close to $-5 / 3$ settles nearly at once at high wave numbers and is stable during the whole process - though the scaling regime again is not very pronounced due to the small Taylor-Reynolds numbers. Therefore, the small scale forcing is strong enough to generate a flow that presents the same characteristics as real three-dimensional turbulence-or also two-dimensional turbulence in the inverse cascade regime. On the other hand, this simulation is not statistically stationary. In Fig. 4(b), we show that there is a difference on a wide range of scales 
between the bubbles forcing term $F_{b}(k)$ and the fluid viscous dissipation $D(k)$. Thus, the condition of stationarity is not fulfilled and the large flow scales are still fed with energy from the small ones.

We again stress that the model for the equation of motion without the lift force does not give a complete representation of the surface forces acting on bubbles or particles of $\mathrm{Re}$ $\sim O(1)$. Indeed, previous works (see e.g., Refs. [17,33] and Ref. [65] for the case of uniform shear) have pointed out the relevance of the lift in this regime. Of course our results can only be expected to be qualitative and not quantitative, as the near-field interactions between the bubbles are not correctly described by the present point-particle approach and as the lift coefficient is set to be constant $C_{L}=1 / 2$. However, refined expressions for the lift force are not likely to give qualitatively different behaviors. The main effect of the lift is to cause the bubble dispersion along the horizontal directions, thus, strongly reducing the anisotropy in the flow caused by the forcing in the vertical direction. Indeed, by definition, the lift force drifts the bubbles in horizontal planes, in directions perpendicular to their average motion.

We now qualitatively investigate the breaking effect of the lift on vertical bubble chains. Note again that for a quantitative analysis the near field as, e.g., obtained from the fronttracking simulations of Refs. [6,7,28,29], is crucial. We base our analysis on the description of the two-bubble long-range interactions proposed in Ref. [52]. The main finding is that a bubble in the wake of another one experiences because of the lift, a lateral force, leading to a deficit of nearby bubbles in the gravity direction (see again Fig. 6).

We further explore this phenomenon by computing the bubble density autocorrelation function defined according to

$$
\rho_{12}(\mathbf{R})=\frac{\left\langle c^{\prime}(\mathbf{x}+\mathbf{R}) c^{\prime}(\mathbf{x})\right\rangle}{\left\langle c^{\prime}(\mathbf{x}) c^{\prime}(\mathbf{x})\right\rangle} .
$$

Here $c^{\prime}(\mathbf{x})$ is the fluctuation of the bubble concentration in $\mathbf{x}$ with respect to the average value $\alpha$ and the brackets denote averages over all $\mathbf{x}$. We consider the autocorrelation in the horizontal $(x-y)$ plane and in the vertical $(z)$ direction separately. The analysis is carried out for the two simulations presented in Fig. 1(b). The results are plotted in Fig. 7. It is shown that, whereas in the simulation without lift forces the pair correlation goes monotonically to zero, in the one with lift the autocorrelation in the $z$ direction becomes negative and later on approaches zero from below. A negative autocorrelation at small distances $R$ is detected also in the horizontal planes. The interpretation of the result is that the bubbles approach is resisted by the lift, and this is occurring especially in the vertical direction, where a bubble rising in the wake of another experiences horizontal forces that expels it from the wake.

Qualitatively, the organization of the bubbles in our simplified simulations is similar to what has been observed in above-mentioned front-tracking simulations by Tryggvason and co-workers [6,7,27-29] or in the experiments of Ref. [22]. Small bubbles with $\operatorname{Re} \sim 1$ were dispersed in a nearly homogeneous manner, with an increasing tendency of horizontal alignment when the bubble Reynolds number approaches 10. Also in the recent experiments by Harteveld $e t$

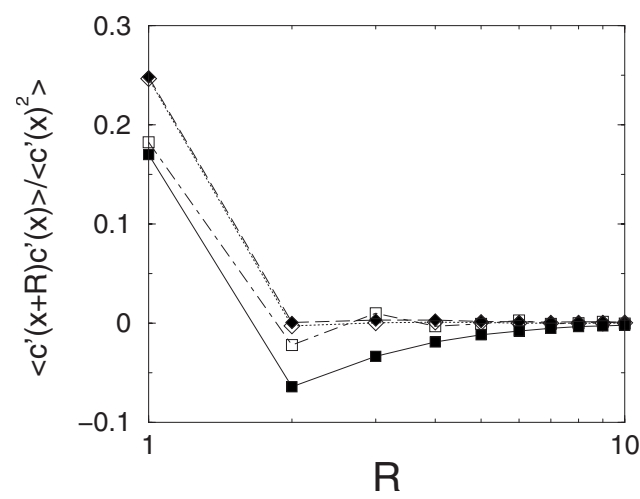

FIG. 7. Autocorrelation function $\rho_{12}(\mathbf{R})$ as a function of the distance $|\mathbf{R}|$, in the horizontal $x$ - $y$ directions (open symbols) and in the vertical $z$ direction (filled symbols). The results indicate simulations without the lift force (diamonds) and with the lift force (squares).

al. [66], bubbly driven flows have been found to be rather homogeneous and no vortex trapping has been detected. The front-tracking simulations show that for even larger bubble Reynolds numbers, horizontal bubble clusters can emerge, which also have been seen in the experiments of [25]. Even in the two-dimensional simulations described in Ref. [67], a preferential tendency of bubbles to stay rather side by side (along the horizontal direction) than in "tandem configuration" (along the vertical) was reported.

\section{CONCLUSIONS}

The behavior of a flow-driven exclusively by rising bubbles has been investigated by direct numerical simulation for the Navier-Stokes equations and Lagrangian tracking for the bubble trajectories, where the bubbles have been treated as point particles on which effective forces act. The evolution of global quantities, such as the total flow energy, as well as of spectral quantities has been followed in time. The results show that the bubble motion initially generates largescale structures by local in scale energy transfer, though the corresponding $-5 / 3$ scaling regime in the spectrum is not very pronounced due to the small Taylor-Reynolds numbers. Later on, however, the bubbles distribution tends to be more disperse, the energy spectrum becomes flat, and energy input equals viscous dissipation at all scales. Therefore, the statistically stationary state of this pseudoturbulent velocity field does not possess the characteristics of real turbulence.

We give qualitative evidence that the physics that determines it are the bubble-bubble indirect interactions that occur via the carrier flow. Indeed, a bubble in the wake of another one, experiences-because of the lift—a horizontal force that prevents the assembling and the stability of vertical clusters, similarly as has been observed in the fronttracking simulations of bubbles of comparable size [27,28] or of larger bubbles [6,7], where the bubbles also distribute homogeneously and horizontal pairs of bubbles are favored. In our case, as a consequence the total forcing induced in the flow is not strong enough to sustain high-energy levels and an inverse energy cascade from small to large scales. 
Does an inverse energy cascade also exist in real (experimental) pseudoturbulence? Also there the energy input is on small scales through the rising bubbles in originally still water and out of this a large-scale motion develops. In this sense, an inverse energy cascade must exist. However, in real pseudoturbulence, also the dissipation in the detailed wake structure of the rising bubbles will play a role. The very recent measurement on the spectra of pseudoturbulence for rising $\mathrm{mm}$ bubbles in Ref. [61] does not show $-5 / 3$ scaling but a scaling exponent consistent with -3 . This latter exponent results from balancing the wake energy input into the flow with dissipation, as suggested by Lance and Bataille [9].

The results presented in this work apply to a flow with periodic boundary conditions. In a real experiment, such as in Taylor-Couette flow, the existence of boundaries can lead to the generation of large-scale vortex structures, which, in turn, affect the bubble motion, as seen in the experiments of Murai et al. [68] and the corresponding numerical simulations of Sugiyama et al. [42]. In those flows, the energy dissipation in the developing boundary layers will play a crucial role in the stationary energy balance.
We understand this work as complementary to the fronttracking simulations. Bunner and Tryggvason [6] ended their numerical study on the dynamics of homogeneous bubble flows with the question: "what happens when the number of bubbles increases beyond 216?" (the number of bubbles they could numerically treat in the paper). Here, we treat up to 288000 bubbles, though in an approximate and simplified way. Nonetheless, we see very similar phenomena as observed in the front-tracking simulations and can even identify the lift force as the origin of the homogeneous bubble distribution by artificially turning it off. A full verification can of course only come from simulations which both resolve the individual bubbles including their wakes and deal with hundred thousands of individual bubbles. Such simulations will, however, unfortunately not be numerically feasible for many years to come.

\section{ACKNOWLEDGMENTS}

The authors thank A. Prosperetti and L. van Wijngaarden for discussions and comments on the paper.
[1] J. Magnaudet and I. Eames, Annu. Rev. Fluid Mech. 32, 659 (2000).

[2] C. T. Crowe, T. Troutt, and J. N. Chung, Annu. Rev. Fluid Mech. 28, 11 (1996).

[3] R. F. Mudde, Annu. Rev. Fluid Mech. 37, 393 (2005).

[4] J. Martinez-Mercado, C. A. Palacios-Morales, and R. Zenit, Phys. Fluids 19, 103302 (2007).

[5] T. H. van den Berg, S. Luther, I. Mazzitelli, J. Rensen, F. Toschi, and D. Lohse, J. Turbul. 7, 1 (2006).

[6] B. Bunner and G. Tryggvason, J. Fluid Mech. 466, 53 (2002).

[7] B. Bunner and G. Tryggvason, J. Fluid Mech. 466, 17 (2002).

[8] L. van Wijngaarden, Theor. Comput. Fluid Dyn. 10, 449 (1998).

[9] M. Lance and J. Bataille, J. Fluid Mech. 222, 95 (1991).

[10] R. F. Mudde, J. S. Groen, and H. E. A. van den Akker, Chem. Eng. Sci. 52, 4217 (1997).

[11] Y. Tsuji and Y. Morikawa, J. Fluid Mech. 120, 385 (1982).

[12] Y. Tsuji, Y. Morikawa, and H. Shiomi, J. Fluid Mech. 139, 417 (1984).

[13] J. M. Rensen, S. Luther, and D. Lohse, J. Fluid Mech. 538, 153 (2005)

[14] T. H. van den Berg, S. Luther, and D. Lohse, Phys. Fluids 18, 038103 (2006).

[15] T. G. Theofanous and J. P. Sullivan, J. Fluid Mech. 116, 343 (1982).

[16] I. Mazzitelli, D. Lohse, and F. Toschi, Phys. Fluids 15, L5 (2003).

[17] I. Mazzitelli, D. Lohse, and F. Toschi, J. Fluid Mech. 488, 283 (2003).

[18] O. A. Druzhinin and S. Elghobashi, Phys. Fluids 10, 685 (1998).

[19] O. A. Druzhinin and S. Elghobashi, J. Fluid Mech. 429, 23 (2001).

[20] M. Maxey, E. Chang, and L. Wang, Exp. Therm. Fluid Sci. 12,
417 (1996).

[21] J. Xu, M. R. Maxey, and G. E. Karniadakis, J. Fluid Mech. 468, 271 (2002).

[22] A. Cartellier and N. Riviere, Phys. Fluids 13, 2165 (2001).

[23] R. F. Mudde and T. Saito, J. Fluid Mech. 437, 203 (2001).

[24] F. Risso and K. Ellingsen, J. Fluid Mech. 453, 395 (2002).

[25] R. Zenit, D. L. Koch, and A. S. Sangani, J. Fluid Mech. 429, 307 (2001).

[26] E. Climent and J. Magnaudet, Phys. Rev. Lett. 82, 4827 (1999).

[27] A. Esmaeeli and G. Tryggvason, J. Fluid Mech. 377, 313 (1998).

[28] A. Esmaeeli and G. Tryggvason, J. Fluid Mech. 385, 325 (1999).

[29] A. Esmaeeli and G. Tryggvason, Phys. Fluids 17, 093303 (2005).

[30] R. N. Parthasarathy and G. M. Faeth, J. Fluid Mech. 220, 485 (1990); 220, 515 (1990).

[31] M. Mizukami, R. N. Parthasarathy, and G. M. Faeth, Int. J. Multiphase Flow 18, 397 (1992).

[32] B. Bunner and G. Tryggvason, Phys. Fluids 11, 1967 (1999).

[33] D. Legendre and J. Magnaudet, J. Fluid Mech. 368, 81 (1998).

[34] G. Sridhar and J. Katz, Phys. Fluids 7, 389 (1995).

[35] A. Tomiyama et al., Chem. Eng. Sci. 57, 1849 (2002).

[36] A. Merle, D. Legendre, and J. Magnaudet, J. Fluid Mech. 532, 53 (2005).

[37] E. Climent and J. Magnaudet, Phys. Fluids 18, 103304 (2006).

[38] E. A. van Nierop, J. J. Bluemink, S. Luther, J. Magnaudet, A. Prosperetti, and D. Lohse, J. Fluid Mech. 571, 439 (2007).

[39] R. Mei, J. Klausner, and C. Lawrence, Phys. Fluids 6, 418 (1994).

[40] J. Magnaudet and D. Legendre, Phys. Fluids 10, 550 (1998).

[41] T. R. Auton, J. Fluid Mech. 183, 199 (1987).

[42] K. Sugiyama, E. Calzavarini, and D. Lohse, J. Fluid Mech. 
608, 21 (2008).

[43] N. H. Thomas, T. R. Auton, K. J. Sene, and J. C. R. Hunt, in Gas Transfer at Water Surfaces, edited by W. Brutsaert and G. H. Jurka (Reidel, Berlin, 1984), p. 255.

[44] J. Hadamard, Acad. Sci., Paris C. R. 152, 1735 (1911).

[45] W. Rybczynski, Bull. Int. Acad. Sci. Cracovie 40, 40 (1911).

[46] M. Maxey and J. Riley, Phys. Fluids 26, 883 (1983).

[47] M. Boivin, O. Simonin, and K. Squires, J. Fluid Mech. 375, 235 (1998).

[48] D. Lohse and F. Toschi, Phys. Rev. Lett. 90, 034502 (2003); E. Calzavarini, D. Lohse, F. Toschi, and R. Tripiccione, Phys. Fluids 17, 055107 (2005).

[49] E. Calzavarini, C. R. Doering, J. D. Gibbon, D. Lohse, A. Tanabe, and F. Toschi, Phys. Rev. E 73, 035301(R) (2006).

[50] L. van Wijngaarden, Appl. Sci. Res. 38, 331 (1982).

[51] L. van Wijngaarden, J. Fluid Mech. 541, 203 (2005).

[52] D. L. Koch, Phys. Fluids A 5, 1141 (1993).

[53] M. P. Brenner, Phys. Fluids 11, 754 (1999).

[54] D. L. Koch and E. S. G. Shaqfeh, J. Fluid Mech. 224, 275 (1991).

[55] H. Nicolai and E. Guazzelli, Phys. Fluids 7, 3 (1995).

[56] H. Nicolai, B. Herzhaft, E. Hinch, L. Oger, and E. Guazzeli, Phys. Fluids 7, 12 (1995).
[57] N. Q. Nguyen and A. J. C. Ladd, J. Fluid Mech. 525, 73 (2005).

[58] Y. Murai, A. Kitagawa, X. Song, J. Ohta, and F. Yamamoto, JSME Int. J., Ser. B 43, 197 (2000).

[59] E. Calzavarini, M. Cencini, D. Lohse, and F. Toschi, Phys. Rev. Lett. 101, 084504 (2008).

[60] E. Calzavarini, M. Kerscher, D. Lohse, and F. Toschi, J. Fluid Mech. 607, 13 (2008).

[61] J. Martínez, D. Chehata, D. P. M. van Gils, C. Sun, and D. Lohse (unpublished).

[62] V. Yakhot and R. Peltz, Phys. Fluids 30, 1272 (1987).

[63] D. Hefer and V. Yakhot, Phys. Fluids A 1, 1383 (1989).

[64] Y. Murai, Y. Matsumoto, X. Song, and F. Yamamoto, JSME Int. J., Ser. B 43, 180 (2000).

[65] D. S. Dandy and H. A. Dwyer, J. Fluid Mech. 216, 381 (1990).

[66] W. K. Harteveld, R. F. Mudde, and H. E. A. van den Akker, Can. J. Chem. Eng. 81, 389 (2003).

[67] A. Esmaeeli and G. Tryggvason, J. Fluid Mech. 314, 315 (1996).

[68] Y. Murai, H. Oiwa, and Y. Takeda, J. Phys.: Conf. Ser. 14, 143 (2005). 